\title{
ON ALMOST-ANALYTIC VECTORS IN ALMOST-KÄHLERIAN MANIFOLDS ${ }^{1)}$
}

\author{
SHUN-ICHI TACHIBANA
}

(Received February 2, 1959)

In pseudo-Kählerian manifolds, many interesting results concerning contravariant or covariant pseudo-analytic vectors are known. ${ }^{2)}$ Even though there were many papers about pseudo-Kählerian manifolds, but were few about almost-Kählerian ones. Recently, M. Apte generalized Bochner's theorem to compact almost-Kählerian manifolds. His work seems to be very suggestive for me. In the present paper we shall generalize several theorems in pseudoKählerian manifolds to almost-Kählerian ones. The main results are integral formulas on vector fields in compact almost-Kählerian manifolds.

In $\S 1$ and $\S 2$ we shall prepare identities and lemmas and in $\$ 3$ and $\$ 4$ define almost-analytic vectors which are generalizations of pseudo-analytic vectors. As applications of integral formulas in $\$ 5$, we shall obtain several theorems in $\$ 6$. In $\$ 7$, we shall give a decomposition theorem of the Lie algebra of contravariant almost-analytic vectors in a compact almost-Kähler-Einstein manifold. The canonical connection will be introduced in $\$ 8$ and in the last section, to contravariant almost-analytic vectors, we shall generalize Apte's theorem.

1. Identities. In an $n$-dimensional real differentiable manifold $M$ with local coordiantes $\left\{x^{i}\right\}$, a tensor field $\varphi_{j}{ }^{i}$ such that

$$
\boldsymbol{\varphi}_{r}{ }^{i} \boldsymbol{\varphi}_{j}^{r}=-\delta_{j}{ }^{i}
$$

is called an almost-complex structure. If an almost-complex structure $\boldsymbol{\varphi}_{j}{ }^{i}$ and a positive definite Riemannian metric tensor $g_{j i}$ on $M$ satisfy the relation

$$
g_{r s} \boldsymbol{\varphi}_{j}{ }^{r} \boldsymbol{\varphi}_{i}{ }^{s}=g_{j i},
$$

then the pair $\left(\boldsymbol{\phi}_{j}{ }^{i}, g_{j i}\right)$ is called an almost-Hermitian structure. Then, from (1.1) and (1.2), we get

$$
\boldsymbol{\phi}_{j i}=-\boldsymbol{\phi}_{i j}
$$

where $\boldsymbol{\phi}_{j i}=\boldsymbol{\varphi}_{j}{ }^{r} g_{r i}$. To an almost-Hermitian structure $\left(\boldsymbol{\varphi}_{j}{ }^{i}, g_{j i}\right)$, an exterior dif-

1) This paper was prepared in a term when the present author was ordered to study at Tohoku University. I wish to express my sincere thanks to Prof. S. Sasaki for his encouragements during the term.

2) For example, cf. Yano, K. [7], Lichnerowicz, A. [3], Sasaki, S. and K. Yano [5], Yano, K. and I. Mogi [9]. 
ferential form $\boldsymbol{\varphi}=\boldsymbol{\varphi}_{j i} d x^{j} \wedge d x^{i}$ can be assoicated. An almost-Hermitian structure is called an almost-Kählerian structure, if the associated differential form $\varphi$ is closed, and then the manifold $M$ is called an almost-Kählerian manifold.

Throughout this paper, by $M$ we shall always mean an $n$-dimensional differentiable manifold with a fixed almost-Kählerian structure $\left(\boldsymbol{\phi}_{j}{ }^{i}, g_{j i}\right)$. In this section, we shall deduce identities which are useful in the later sections.

In our $M$, the form $\varphi$ being closed, so we have

$$
\nabla_{k} \boldsymbol{\varphi}_{j h}+\nabla_{j} \boldsymbol{\varphi}_{i k}+\nabla_{i} \boldsymbol{\varphi}_{k j}=0
$$

where $\nabla k$ denotes the operator of covariant derivative with respect to the Riemannian connection.

On the other hand, since the identity

$$
\nabla^{r} \boldsymbol{\varphi}_{r h}=-\frac{3}{2} \nabla_{[r} \boldsymbol{\varphi}_{p q]} \boldsymbol{\varphi}^{r p} \boldsymbol{\varphi}_{h}{ }^{q}
$$

is well known ${ }^{4}$, in our case, we have

$$
\nabla^{r} \boldsymbol{\varphi}_{r h}=0 .
$$

The Nijenhuis' tensor $N_{j i}{ }^{h}$ of an almost-complex structure $\boldsymbol{\varphi}_{j}{ }^{i}$ is defined by

$$
N_{j i}{ }^{h}=\boldsymbol{\varphi}_{j}{ }^{l}\left(\nabla_{l} \boldsymbol{\varphi}_{i}{ }^{h}-\nabla_{i} \boldsymbol{\varphi}_{l}{ }^{h}\right)-\boldsymbol{\varphi}_{i}{ }^{l}\left(\nabla_{l} \boldsymbol{\varphi}_{j}{ }^{h}-\nabla_{j} \boldsymbol{\varphi}_{l}{ }^{h}\right),
$$

so on taking account of (1.1), (1.3) and (1.4), we find

$$
N_{j i}{ }^{h}=2 \boldsymbol{\varphi}_{j}{ }^{l}\left(\nabla_{l} \boldsymbol{\varphi}_{i}{ }^{h}-\nabla_{i} \boldsymbol{\varphi}_{l}{ }^{h}\right) .
$$

Let $R_{k j i}{ }^{h}$ be Riemannian curvature tensor, that is,

$$
R_{k j i}{ }^{h}=\partial_{k}\left\{\begin{array}{l}
h \\
j i
\end{array}\right\}-\partial_{j}\left\{\begin{array}{c}
h \\
k i
\end{array}\right\}+\left\{\begin{array}{c}
h \\
k r
\end{array}\right\}\left\{\begin{array}{c}
r \\
j i
\end{array}\right\}-\left\{\begin{array}{c}
h \\
j r
\end{array}\right\}\left\{\begin{array}{c}
r \\
k i
\end{array}\right\}
$$

where $\partial_{k}=\partial / \partial x^{k}$, and put

and

$$
R_{j i}=R_{r j i}^{r}, \quad R_{k j i h}=R_{k j i}^{r} g_{r h}
$$

$$
R_{k j}^{*}=\frac{1}{2} \varphi^{p q} R_{p q r j} \varphi_{k}^{r}
$$

We notice that, in pseudo-Kählerian manifold, $R_{k j}^{*}=R_{k j}$ holds good. The Ricci's identities are given by the following formulas for any vector field $v_{i}$ and $v^{i}$.

$$
\begin{aligned}
& \nabla_{k} \nabla_{j} v_{i}-\nabla_{j} \nabla_{k} v_{i}=-R_{k j i}{ }^{r} v_{r}, \\
& \nabla_{k} \nabla_{j} v^{i}-\nabla_{j} \nabla_{k} v^{i}=R_{k j r}{ }^{i} v^{r} .
\end{aligned}
$$

Applying to $\boldsymbol{\varphi}_{i}{ }^{h}$ the Ricci's identity, we have

$$
\nabla_{k} \nabla_{j} \boldsymbol{\varphi}_{i}{ }^{h}-\nabla_{j} \nabla_{k} \boldsymbol{\varphi}_{i}{ }^{h}=R_{k j r}{ }^{h} \boldsymbol{\varphi}_{i}{ }^{r}-R_{k j i}{ }^{r} \boldsymbol{\varphi}_{r}{ }^{h} .
$$

3) Indices $i, j, k, \cdots \cdots p, q, r, s, \cdots \cdot$ run over $1, \cdots \cdots, n$. Notations are followed to Yano, $\mathrm{K}$. [7] except some trivial changes.

4) Schouten, J. A. and K. Yano [6].

5) For example Yano, K. [7], p. 229 
Transvecting the last equation with $g^{k i}$ and using (1.5), we find

$$
\nabla^{r} \nabla_{j} \boldsymbol{\varphi}_{r}{ }^{h}=R_{i j r}{ }^{h} \boldsymbol{\varphi}^{i r}+R_{j}^{r} \boldsymbol{\varphi}_{r}{ }^{h},
$$

where we put $\phi^{i r}=\phi_{p}^{r} g^{p_{i}}$. On the other hand, $\boldsymbol{\phi}^{i r}$ being skew symmetric with respect to $i$ and $r$, so we have

$$
R_{i j r}{ }^{h} \boldsymbol{\phi}^{i r}=\frac{1}{2} \boldsymbol{\varphi}^{i r}\left(R_{i j r}{ }^{h}-R_{r j i}{ }^{h}\right)=\frac{1}{2} \boldsymbol{\phi}^{r i} R_{r i j}{ }^{h} .
$$

Hence, it follows that

$$
\nabla^{r} \nabla_{j} \boldsymbol{\varphi}_{r}{ }^{h}=\frac{1}{2} \boldsymbol{\varphi}^{p q} R_{p q j}{ }^{h}+R_{j}^{r} \boldsymbol{\varphi}_{r}{ }^{h},
$$

and from which we obtain

$$
\nabla^{r} \nabla_{j} \boldsymbol{\varphi}_{r h}=\frac{1}{2} \boldsymbol{\phi}^{p q} R_{p q j h}+R_{j}^{r} \boldsymbol{\varphi}_{r h} .
$$

$$
\text { Operating } \begin{aligned}
\nabla^{k}=g^{k r} \nabla_{r} \quad \text { to (1.4), we have } \\
\nabla^{k} \nabla_{k} \boldsymbol{\varphi}_{j i}=\nabla^{k} \nabla_{j} \boldsymbol{\varphi}_{k i}-\nabla^{k} \nabla_{i} \boldsymbol{\varphi}_{k j} .
\end{aligned}
$$

Hence on taking account of (1.8) we find

$$
\nabla^{r} \nabla_{r} \boldsymbol{\varphi}_{j i}=\boldsymbol{\varphi}^{p q} R_{p q j i}+R_{j}^{r} \boldsymbol{\varphi}_{r i}-R_{i}^{r} \boldsymbol{\varphi}_{r j}
$$

The equations (1.8) and (1.9) are important identities in the later sections.

By a vector field $v$ we always mean a contravariant vector field $v^{i}$, a covariant vector field $v_{i}=g_{i r} v^{r}$ and a differential form $v=v_{i} d x^{i}$. So the word "a vector is closed" means that the corresponding form $v$ is closed.

Let $v$ be any vector field, then from (1.3) and Ricci's identity we get

$$
\boldsymbol{\phi}^{p q} \nabla p \nabla q v_{i}=-\frac{1}{2} \boldsymbol{\phi}^{p q} R_{p q i r} v^{r}
$$

And from (1.8) and (1.10) we obtain

$$
\boldsymbol{\varphi}_{l}{ }^{i} \boldsymbol{v}^{r} \nabla^{j} \nabla r \boldsymbol{\varphi}_{j i}=-\boldsymbol{v}^{r} R_{r l}^{*}+\boldsymbol{v}^{r} R_{r l},
$$

$$
\boldsymbol{\varphi}_{l}{ }^{i} \boldsymbol{\varphi}^{j r} \nabla_{j} \nabla_{r} \boldsymbol{v}_{i}=-\boldsymbol{v}^{r} R_{l r}^{*},
$$

where $R_{l r}^{*}$ is given by (1.7).

From (1.3) and (1.4) we have

$$
\boldsymbol{\phi}_{h}^{j} \boldsymbol{\phi}_{s}^{r}\left(\nabla^{h} \boldsymbol{\phi}^{s t}\right) \boldsymbol{\varphi}_{t}{ }^{i}=\boldsymbol{\varphi}_{h}{ }^{j} \nabla^{h} \boldsymbol{\phi}^{i r}=-\boldsymbol{\varphi}_{h}{ }^{j}\left(\nabla^{i} \boldsymbol{\phi}^{r h}+\nabla^{r} \boldsymbol{\phi}^{h i}\right)
$$

Interchanging $r$ and $j$ and the adding the equation thus obtained to the last equation, we get

$$
\left(\nabla^{j} \boldsymbol{\phi}^{r t}+\nabla^{r} \boldsymbol{\phi}^{j t}\right) \boldsymbol{\varphi}_{t}{ }^{i}=-\boldsymbol{\phi}_{h}{ }^{j} \boldsymbol{\phi}_{s}{ }^{r}\left(\nabla^{h} \boldsymbol{\phi}^{s t}+\nabla^{s} \boldsymbol{\phi}^{h t}\right) \boldsymbol{\varphi}_{t}{ }^{i}
$$

Consequently, for any vector field $v$ the following equation holds good. 


$$
\nabla_{j} \boldsymbol{v}_{r}\left(\nabla^{j} \boldsymbol{\phi}^{r_{t}}+\nabla^{r} \boldsymbol{\phi}^{j t}\right) \boldsymbol{\phi}_{t}{ }^{i}=-\boldsymbol{\phi}_{h}{ }^{j} \boldsymbol{\phi}_{s}{ }^{r}\left(\nabla_{j} \boldsymbol{v}_{r}\right)\left(\nabla^{h} \boldsymbol{\phi}^{s t}+\nabla^{s} \boldsymbol{\phi}^{h t}\right) \boldsymbol{\phi}_{t}{ }^{i} .
$$

2. Lemmas. For convenience sake, we shall expose several lemmas which are well known. By $V_{n}$ we shall always mean an $n$-dimensional Riemannian manifold.

LEMMA 2.1.6) In a compact, orientable $V_{n}$, the following integral formulas are valid for any vector field $v$.

$$
\begin{aligned}
& \int_{V_{n}}\left[\left(\nabla^{r} \nabla_{r} v_{i}-R_{r i} v^{r}\right) v^{i}+S(v)\right] d \sigma=0, \\
& \int_{V_{n}}\left[\left(\nabla^{r} \nabla_{r} v_{i}+R_{r i} v^{r}\right) v^{i}+T(v)\right] d \sigma=0,
\end{aligned}
$$

where $d \sigma$ means the volume element of the $V_{n}$, and $S(v)$ and $T(v)$ are defined by

$$
\begin{aligned}
& S(v)=\frac{1}{2}\left(\nabla^{s} v^{r}-\nabla^{r} v^{s}\right)\left(\nabla_{s} v_{r}-\nabla_{r} v_{s}\right)+\left(\nabla^{r} v_{r}\right)\left(\nabla^{s} v_{s}\right), \\
& T(v)=\frac{1}{2}\left(\nabla^{s} v^{r}+\nabla^{r} v^{s}\right)\left(\nabla_{s} v_{r}+\nabla_{r} v_{s}\right)-\left(\nabla^{r} v_{r}\right)\left(\nabla^{s} v_{s}\right)
\end{aligned}
$$

respectively.

In a $V_{n}$, a vector field $v$ is called a Killing vector (or an infinitesimal isometry) if it satisfies

$$
\underset{v}{\stackrel{2}{J}} g_{j i} \equiv \nabla_{j} v_{i}+\nabla_{i} v_{j}=0
$$

where ${\underset{v}{v}}_{v}$ denotes the operator of Lie differentiation with respect to $v^{i}$. For Killing vectors, the following theorem is well known.

LEMMA 2. 2. ${ }^{7)}$ In a compact, orientable $V_{n}$, a necessary and sufficient condition for $v$ to be a Killing vector is that

$$
\nabla^{r} \nabla_{r} v_{i}+R_{r i} v^{r}=0, \quad \nabla^{r} v_{r}=0 .
$$

In a $V_{n}$, a vector field $v$ is called a conformal Killing vector (or an infinitesimal conformal transformation) if it satisfies

$$
\underset{v}{\stackrel{f}{J}} g_{j i} \equiv \nabla_{j} v_{i}+\nabla_{i} v_{j}=2 \phi g_{j i}
$$

where $\phi$ is a scalar function. Then as is well known, it holds the following

LEMMA 2.3. ${ }^{8)}$ In a compact, orientable $V_{n}$, a necessary and sufficient condition in order that $v$ be a conformal Killing vector is that

6) Fo: example, Yano, K. [7], p. 278.

7) For example, Yano, K. [7], p. 221.

8) Yano, K. [7], p. 278. 


$$
\nabla^{r} \nabla_{r} v_{i}+R_{r i} v^{r}+\frac{n-2}{n} \nabla_{i} \nabla_{r} v^{r}=0 .
$$

In a $V_{n}$, a vector field $v$ is called a projective Killing vector ${ }^{9)}$ if it satisfies

$$
\underset{v}{\mathcal{L}}\left\{{ }_{j i}^{h}\right\} \equiv \nabla_{j} \nabla_{i} v^{h}+R_{r j i}{ }^{h} v^{r}=\delta_{j}{ }^{h} \psi_{i}+\delta_{i}{ }^{h} \psi_{j}
$$

$\psi_{i}$ being a certain vector field. Transvecting (2.6) with $g^{j i}$, we get

$$
\nabla^{r} \nabla_{r} v^{h}+R_{r}{ }^{h} v^{r}=2 \psi^{h} .
$$

By contraction with respect to $h$ and $i$ in (2.6), it follows that

$$
\nabla j \nabla r v^{r}=(n+1) \psi_{j} .
$$

From (2.7) and (2.8), we have

$$
\nabla^{r} \nabla_{r} v_{i}+R_{r i} v^{r}=\frac{2}{n+1} \nabla_{i} \nabla_{r} v^{r}
$$

for a projective Killing vector $v$.

Since an almost-Kählerian manifold $M$ is an orientable Riemannian manifold, the above lemmas and arguments are valid for our $M$.

For a vector field $v$ we define $\widetilde{v}$ by

$$
\widetilde{v_{i}}=\boldsymbol{\varphi}_{i}{ }^{t} v_{t}, \quad \widetilde{v^{i}}=g^{i r} v_{r}=-\boldsymbol{\varphi}_{t}{ }^{i} v_{t},
$$

then we have

and

$$
\nabla_{r}{\widetilde{v_{i}}}=\left(\nabla_{r} \boldsymbol{\varphi}_{i}{ }^{t}\right) \boldsymbol{v}_{t}+\boldsymbol{\varphi}_{i}{ }^{t} \nabla_{r} \boldsymbol{v}_{t}
$$

$$
\nabla^{r} \nabla_{r} \widetilde{v}_{i}=\left(\nabla^{r} \nabla_{r} \boldsymbol{\varphi}_{i}{ }^{t}\right) \boldsymbol{v}_{t}+2\left(\nabla^{r} \boldsymbol{\varphi}_{i}{ }^{t}\right) \nabla_{r} \boldsymbol{v}_{t}+\boldsymbol{\varphi}_{i}{ }^{t} \nabla^{r} \nabla_{r} \boldsymbol{v}_{t}
$$

Substituting (1.9) in the right hand side of the last equation, we get

$$
\begin{aligned}
\nabla^{r} \nabla r \widetilde{v}_{i} & =\left(\varphi^{p q} R_{p q i t}+R_{i}^{r} \boldsymbol{\varphi}_{r t}-R_{t}^{r} \boldsymbol{\varphi}_{r i}\right) \boldsymbol{v}^{t} \\
& +2\left(\nabla^{r} \boldsymbol{\varphi}_{i}{ }^{t}\right) \nabla_{r} \boldsymbol{v}_{t}+\boldsymbol{\varphi}_{i}{ }^{t} \nabla^{r} \nabla_{r} \boldsymbol{v}_{t},
\end{aligned}
$$

from which we find

$$
\begin{aligned}
\left(\nabla^{r} \nabla r \widetilde{v_{i}}\right. & \left.-R_{r i} \widetilde{v^{r}}\right) \widetilde{v^{i}}=\left(\nabla^{r} \nabla_{r} v_{i}+R_{r i} v^{r}\right) v^{i} \\
& -2 R_{r i}^{*} v^{r} v^{i}+2\left(\nabla^{r} v^{t}\right)\left(\nabla_{r} \boldsymbol{\varphi}_{t i}\right) \boldsymbol{\phi}_{p}^{i} \boldsymbol{v}^{p} .
\end{aligned}
$$

On the other hand, by virtue of (1.2) and (1.4), we find

$$
2 \nabla_{r} \boldsymbol{\varphi}_{t i}=\nabla_{r} \boldsymbol{\varphi}_{t i}+\nabla_{t} \boldsymbol{\varphi}_{r i}-\nabla_{i} \boldsymbol{\varphi}_{r t},
$$

so $(2,11)$ can be written as

$$
\begin{aligned}
\left(\nabla^{r} \nabla_{r} \widetilde{v_{i}}-R_{r i} \widetilde{v^{i}}\right) \widetilde{v^{r}} & =\left(\nabla^{r} \nabla_{r} v_{i}+R_{r i} v^{r}\right) v^{i} \\
& +\nabla^{r} v^{t}\left(\nabla_{r} \boldsymbol{\varphi}_{t i}+\nabla_{t} \boldsymbol{\varphi}_{r i}\right) \boldsymbol{\varphi}_{p}{ }^{i} \boldsymbol{v}^{p}
\end{aligned}
$$

9) Yano, K. [7], p. 133. 


$$
-\left\{2 R_{r p}^{*} v^{r}+\nabla^{r} v^{t}\left(u_{i} \boldsymbol{\Phi}_{r t}\right) \boldsymbol{\Phi}_{p}{ }^{i}\right\} \boldsymbol{v}^{p}
$$

Hence, if the equation

$$
\nabla^{r} \boldsymbol{v}^{t}\left(\nabla_{r} \boldsymbol{\varphi}_{t i}+\nabla_{t} \boldsymbol{\varphi}_{r i}\right) \boldsymbol{\varphi}_{p}{ }^{i} \boldsymbol{v}^{p}=0
$$

holds, by virtue of (2.12) and lemma 2.1, we get the following

LEMMA 2.4. In a compact, almost-Kählerian manifold $M$, if the equation (2.13) holds, the integral formula

$$
\left.\int_{M}\left(\nabla^{r} \nabla_{r} v_{i}+R_{r i} v^{r}\right) v^{i}-\left\{2 R_{r p}^{*} v^{r}+\nabla^{r} v^{t}\left(\nabla_{i} \boldsymbol{\varphi}_{r t}\right) \boldsymbol{\varphi}_{p}{ }^{j}\right\} \boldsymbol{v}^{p}+S(\widetilde{\boldsymbol{v}})\right] d \sigma=0
$$

is valid, where $S(\widetilde{v})$ is given by

$$
\begin{aligned}
& S(\widetilde{v})=\frac{1}{2}\left(\nabla^{r} \widetilde{v^{s}}-\nabla^{s} \widetilde{v^{r}}\right)\left(\nabla_{r} \widetilde{v_{s}}-\nabla_{s} \widetilde{v_{r}}\right)+\left(\nabla^{r} \widetilde{v_{r}}\right)\left(\nabla^{s} \widetilde{v_{s}}\right), \\
& \widetilde{v_{i}}=\phi_{i}{ }^{t} v_{t} .
\end{aligned}
$$

3. Contravariant almost-analytic vectors. In this section, we shall generalize a notion of contravariant pseudo-analytic vectors ${ }^{10)}$ in pseudoKählerian manifold to an almost-Kählerian manifold $M$.

In our manifold $M$, we shall say that a vector field $v$ is contravariant almost-analytic (or analytic) if it satisfies

$$
\underset{v}{\stackrel{f}{L}} \boldsymbol{\varphi}_{j}{ }^{i} \equiv \boldsymbol{v}^{r} \nabla_{r} \boldsymbol{\varphi}_{j}{ }^{i}-\boldsymbol{\varphi}_{j}{ }^{r} \nabla r v^{i}+\boldsymbol{\varphi}_{r}{ }^{i} \nabla_{j} v^{r}=0 \text {. }
$$

In the next section, we shall define a covariant almost-analytic vector, but by analytic vectors we shall always mean contravariant almost-analytic vectors.

Let $v$ be an analytic vector, then from (3.1), we have

$$
v^{r} \nabla_{r} \boldsymbol{\phi}_{j i}=\boldsymbol{\phi}_{j}^{r} \nabla_{r} v_{i}+\boldsymbol{\phi}_{i}^{r} \nabla_{j} v_{r}
$$

from which, by virtue of (1.4), we find

$$
\nabla_{j} \widetilde{v_{i}}-\nabla_{i} \widetilde{v_{j}}=-\boldsymbol{\varphi}_{j}{ }^{r}\left(\nabla_{r} v_{i}+\nabla_{i} v_{r}\right) \text {. }
$$

These three equations are equivalent to each other.

From (3.3) we obtain

THEOREM 3.1. In an almost-Kählerian manifold, a necessary and sufficient condition in order that an analytic vector $v$ be a Killing vector is that the vector $\widetilde{v}$ is closed, where $\widetilde{v}_{i}=\varphi_{i}{ }^{t} v_{t}$.

From (3.2), for an analytic vector $v$ it holds that

$$
\left(\nabla^{j} v^{r}\right) \nabla_{r} \boldsymbol{\varphi}_{j i}+v^{r} \nabla^{j} \nabla_{r} \boldsymbol{\varphi}_{j i}-\boldsymbol{\varphi}_{j}{ }^{r} \nabla^{j} \nabla_{r} v_{i}-\left(\nabla^{j} \boldsymbol{\phi}_{i}{ }^{r}\right) \nabla_{j} v_{r}-\boldsymbol{\varphi}_{i}{ }^{r} \nabla^{j} \nabla_{j} v_{r}=0 .
$$

Transvecting the last equation with $\boldsymbol{\phi}_{l}{ }^{i}$ we get

10) Yano, K. [7], p. 236. 


$$
\nabla^{r} \nabla \boldsymbol{r} \boldsymbol{v}_{l}+\boldsymbol{\varphi}_{l}{ }^{i} \boldsymbol{v}^{r} \nabla^{j} \nabla_{r} \boldsymbol{\varphi}_{j i}-\boldsymbol{\varphi}_{l}{ }^{i} \boldsymbol{\phi}^{j r} \nabla_{j} \nabla r \boldsymbol{v}_{i}+\nabla^{j} \boldsymbol{v}^{r}\left(\nabla_{j} \boldsymbol{\varphi}_{r_{i}}+\nabla_{r} \boldsymbol{\Phi}_{j i}\right) \boldsymbol{\varphi}_{p}{ }^{i} \boldsymbol{v}^{p}=0 .
$$

On taking account of (1.11) and (1.12), the above equation can be written in the following form,

$$
\nabla^{r} \nabla r v_{i}+R_{r i} v^{r}+\nabla^{j} v^{r}\left(\nabla_{j} \boldsymbol{\varphi}_{r t}+\nabla_{r} \boldsymbol{\varphi}_{j t}\right) \boldsymbol{\phi}_{i}{ }^{t}=0 .
$$

On the other hand, from (3.2) we have

$$
\nabla_{j} v_{r}-\boldsymbol{\varphi}_{j}^{h} \boldsymbol{\varphi}_{r}^{s} \nabla_{h} \boldsymbol{v}_{s}=-\boldsymbol{v}^{s}\left(\nabla_{s} \boldsymbol{\varphi}_{j t}\right) \boldsymbol{\varphi}_{r}{ }^{t}
$$

Hence for an analytic vector $v$ we obtain, from (1.13) and (3.5),

$$
\nabla_{j} v_{r}\left(\nabla^{j} \boldsymbol{\phi}^{r t}+\nabla^{r} \boldsymbol{\phi}^{j t}\right) \boldsymbol{\varphi}_{t}{ }^{i}=0
$$

because the right hand side of (3.5) is skew symmetric with respect to $j$ and $r$. Consequently we find

$$
\nabla^{r} \nabla_{r} v_{i}+R_{r i} v^{r}=0
$$

for any analytic vector field $v$. The equation (3.7) can be obtained in the following way, also. For an analytic vector, we have $\underset{v}{\mathscr{f}} \boldsymbol{\varphi}_{j}{ }^{i}=0$. Hence from (1.6) it holds that

$$
\frac{1}{2} \underset{v}{\stackrel{f}{v}} N_{j i}{ }^{h}=\boldsymbol{\varphi}_{j}{ }^{l}\left(\underset{v}{\stackrel{f}{j}} \nabla_{l} \boldsymbol{\varphi}_{i}{ }^{h}-\underset{v}{\mathcal{f}} \nabla_{i} \boldsymbol{\varphi}_{l}{ }^{h}\right) .
$$

On the other hand, the following formula holds good,

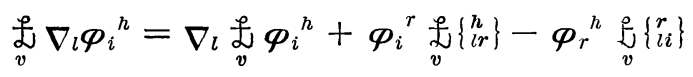

between Lie differentiation and covariant derivative. ${ }^{12)}$ Hence, it follows that

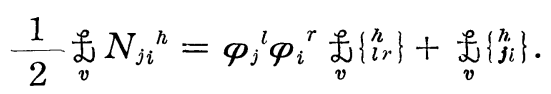

Transvecting the last equation with $g^{j i}$ and taking account of skew symmetry of $N_{j i}{ }^{h}$, we get

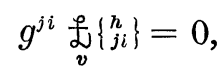

from which we get again the equation (3.7).

4. Covariant almost-analytic vectors. In a pseudo-Kählerian manifold, a vector field $v$ is called a covariant pseudo-analytic vector ${ }^{13)}$ if it satisfies

$$
\boldsymbol{\phi}_{j}^{r} \nabla_{i} \boldsymbol{v}_{r}-\boldsymbol{\phi}_{i}^{r} \nabla_{r} \boldsymbol{v}_{j}=0 \text {. }
$$

If the manifold is compact, any covariant pseudo-analytic vector coincides with a harmonic vector. ${ }^{14)}$ And the following theorem is well known. ${ }^{15)}$

12) Yano, K. [7], p. 16.

13) Yano, K. [7], p. 235.

14) Yano, K. [7], p. 236.

15) Sasaki, S. and K. Yano [5]. 
In a compact pseudo-Kählerian manifold, the inner product of a contravariant pseudo-analytic vector and a covariant pseudo-analytic vector is constant over the manifold.

In this section we shall define a covariant almost-analytic vector in almost-Kählerian manifolds so that an analogous theorem holds good if the manifold is compact.

In the first place, we shall prove the following Lemma which is a generalization of Liouvilles theorem in the theory of functions of a complex variable.

LEMMA 4.1. In a compact almost-Kählerian manifold $M$, if scalar functions $f$ and $g$ satisfy the equation

$$
\nabla_{i} f=\boldsymbol{\varphi}_{i}^{r} \nabla_{r} g
$$

then the functions are both constant over the $M$.

PROOF. From (4.1) we have $\nabla^{i} \nabla_{i} f=\phi^{i r} \nabla_{i} \nabla_{r} g=0$, from which it follows that

$$
\nabla\left(f^{2}\right) \equiv \nabla^{i} \nabla_{i}\left(f^{2}\right)=2\left(\nabla^{i} f\right)\left(\nabla_{i} f\right) .
$$

Hence, by Green's theorem we get $\nabla_{i} f=0$. q. e. d.

Let $u$ be a vector field and $v$ be an analytic vector and put $g=u_{\imath} v^{l}$. Then

$$
\boldsymbol{\varphi}_{i}{ }^{r} \nabla_{r} g=\boldsymbol{\varphi}_{i}{ }^{r}\left(\nabla_{r} u_{l}\right) \boldsymbol{v}^{l}+\boldsymbol{\varphi}_{i}{ }^{r}\left(\nabla_{r} \boldsymbol{v}^{l}\right) u_{l} \text {. }
$$

Since $v$ is an analytic vector, by virtue of (2.1), we have

$$
\boldsymbol{\varphi}_{i}^{r} \nabla_{r} v^{l}=v^{r} \nabla_{r} \boldsymbol{\varphi}_{i}{ }^{l}+\boldsymbol{\varphi}_{r}{ }^{l} \nabla_{i} v^{r} \text {. }
$$

Substituting this into, (4.2), we get

$$
\boldsymbol{\phi}_{i}{ }^{r} \nabla_{r} g=\boldsymbol{\varphi}_{r}{ }^{l} u_{l} \nabla_{i} v^{r}+\left(u_{l} \nabla_{r} \boldsymbol{\varphi}_{i}{ }^{l}+\boldsymbol{\varphi}_{i}{ }^{l} \nabla_{l} u_{r}\right) v^{r} \text {. }
$$

Next we put $f=\varphi_{r}{ }^{l} u_{\imath} v^{r}$, then it follows that

$$
\nabla_{i} f=\varphi_{r}{ }^{l} u_{l} \nabla_{i} v^{r}+\nabla_{i}\left(\boldsymbol{\varphi}_{r}{ }^{l} u_{l}\right) v^{r} .
$$

Comparing (4.3) with (4.4), if the vector $u$ satisfies the equation

$$
\nabla_{i}\left(\phi_{r}{ }^{l} u_{l}\right)=u_{l} \nabla_{r} \boldsymbol{\varphi}_{i}{ }^{l}+\boldsymbol{\phi}_{i}{ }^{l} \nabla_{l} u_{r},
$$

i. e.

$$
\nabla_{i}\left(\boldsymbol{\varphi}_{j}{ }^{r} u_{r}\right)=u_{r} \nabla_{j} \boldsymbol{\varphi}_{i}{ }^{r}+\boldsymbol{\varphi}_{i}{ }^{r} \nabla_{r} u_{j},
$$

we find that the functions $f$ and $g$ defined above satisfy (4.1).

We call $u$ satisfying (4.5) a covariant almost-analytic vector (or a covariant analytic vector).

This vector is a generalization of a covariant pseudo-analytic vector in a pseudo-Kählerian manifold.

By the above definition and Lemma 4.1 we have the following 
THEOREM 4.2. In a compact, almost-Kählerian manifold, the inner product of an analytic vector and a covariant analytic vector is constant over $M$.

We shall deform the equation (4.5) which gives the definition of a covariant analytic vector. From (4.5), we have

$$
u^{r}\left(-\nabla_{j} \boldsymbol{\varphi}_{i r}+\nabla_{i} \boldsymbol{\varphi}_{j r}\right)=\boldsymbol{\varphi}_{i}^{r} \nabla_{r} u_{j}-\boldsymbol{\varphi}_{j}^{r} \nabla_{i} u_{r}
$$

On taking account of (1.4) we find

$$
u^{r} \nabla_{r} \boldsymbol{\varphi}_{j i}=\phi_{i}^{r} \nabla_{r} u_{j}-\phi_{j}^{r} \nabla_{i} u_{r} .
$$

As the left hand side is skew symmetric with respect to $j$ and $i$, we get

$$
u^{r} \nabla_{r} \boldsymbol{\varphi}_{j i}=-\boldsymbol{\phi}_{j}^{r} \nabla_{r} u_{i}+\boldsymbol{\phi}_{i}^{r} \nabla_{j} u_{r} .
$$

We call our attention to the similarity of (3.2) and (4.7).

On the otherhand, from (4.5) we have

$$
\nabla_{i}\left(\boldsymbol{\varphi}_{j}{ }^{r} u_{r}\right)=\nabla_{j}\left(\boldsymbol{\varphi}_{i}{ }^{r} u_{r}\right)-\boldsymbol{\varphi}_{i}{ }^{r} \nabla_{j} u_{r}+\boldsymbol{\varphi}_{i}{ }^{r} \nabla_{r} u_{j},
$$

from which we find

$$
\nabla_{i} \widetilde{u}_{j}-\nabla_{j} \widetilde{u}_{i}=\phi_{i}{ }^{r}\left(\nabla_{r} u_{j}-\nabla_{j} u_{r}\right)
$$

The equations (4.5), (4.6), (4.7) and (4.8) are equivalent to each other. Transvecting (4.5) with $g^{j i}$, the equation

$$
\nabla^{r} \tilde{u_{r}}=0
$$

holds good for a covariant analytic vector $u$. From (4.8) and (4.9), we have

THEOREM 4.3. In an almost-Kählerian manifold $M$, if a covariant analytic vector $u$ is closed, then $\tilde{u}$ is harmonic.

In general, $\tilde{v}$ is not necessarily a harmonic resp. analytic vector even though $v$ is a harmonic resp. analytic vector, $l u t$ about covariant analytic vectors we have the following

THEOREM 4.4. In an almost-Kählerian manifold $M$, if a vector field $u$ is a covariant analytic vector, then $\widetilde{u}$ is covariant analytic.

PROOF. We put $v_{i}=\widetilde{u}_{i}=\varphi_{i}{ }^{t} u_{t}$, then $\widetilde{v}_{i}=-u_{i}$. From (4.8) we have

$$
\nabla_{i} v_{j}-\nabla_{j} v_{i}=-\phi_{i}{ }^{r}\left(\nabla_{r} \widetilde{v}_{j}-\nabla_{j} \widetilde{v}_{r}\right) \text {. }
$$

Transvecting the last equation with $\boldsymbol{\varphi}_{t}{ }^{j}$, we find

$$
\boldsymbol{\varphi}_{t}{ }^{i}\left(\nabla_{i} \boldsymbol{v}_{j}-\nabla_{j} \boldsymbol{v}_{i}\right)=\nabla_{t} \widetilde{v}_{j}-\nabla_{j} \widetilde{v}_{t} . \quad \text { q. e.d. }
$$

From (4.8), we have evidently the following

THEOREM 4.5. In an almost-Kählerian manifold $M$, if vector fields $u$ and $\tilde{u}$ are both closed, then $u$ is a covariant analytic vector. 
Transvecting (4.7) with $\boldsymbol{\varphi}_{l}{ }^{j}$ it follows that

$$
u^{r}\left(\nabla_{r} \boldsymbol{\varphi}_{j i}\right) \boldsymbol{\varphi}_{l}{ }^{j}=-\left(\boldsymbol{\varphi}_{l}^{j} \boldsymbol{\varphi}_{j}^{r}+\delta_{l}{ }^{j} \boldsymbol{\delta}_{j}{ }^{r}\right) \nabla_{r} u_{i}
$$

The left hand side is skew symmetric with respect to $j$ and $l$, so find

THEOREM 4.6. In an almost-Kählerian manifold $M$, if a covariant analytic vector is closed, then we have

$$
u^{r} \nabla_{r} \boldsymbol{\varphi}_{j i}=0 .
$$

In $\$ 6$, we shall prove that a covariant analytic vector is always closed if the manifold $M$ is compact.

If a vector field $v$ is at the same time analytic and covariant analytic, then we have $\nabla_{j} v_{i}=0$, by virtue of (3.2) and (4.7). Hence

THEOREM 4.7.16) In an almost-Kählerian manifold $M$, if an analytic vector is at the same time covariant analytic, then it is a parallel vector field.

Let $u$ be a covariant analytic vector, then from (4.7) we have

$$
\nabla^{r} \nabla_{r} \boldsymbol{u}_{l}+\boldsymbol{\varphi}_{l}{ }^{i} u^{r} \nabla^{j} \nabla_{r} \boldsymbol{\varphi}_{j i}+\boldsymbol{\varphi}_{l}{ }^{i} \boldsymbol{\phi}^{j r} \nabla_{j} \nabla_{r} \boldsymbol{u}_{i}+\nabla^{j} u^{r}\left(\nabla_{r} \boldsymbol{\varphi}_{j i}+\nabla_{j} \boldsymbol{\varphi}_{r i}\right) \boldsymbol{\varphi}_{l}{ }^{i}=0 .
$$

On taking account of (1.11) and (1.12), we find

$$
\nabla^{r} \nabla_{r} u_{l}+R_{r l} u^{r}-R_{r l}^{*} u^{r}+\nabla^{j} u^{r}\left(\nabla_{j} \boldsymbol{\varphi}_{r i}+\nabla_{r} \boldsymbol{\varphi}_{j i}\right) \boldsymbol{\phi}_{l}{ }^{i}=0 .
$$

5. Integral formulas. In the present section, we shall prove some formulas on vector fields in a compact almost-Kählerian manifold.

Consider a vector field $v$ in an almost-Kählerian manifold $M$ and define tensors $a(v)_{j k}$ and $b(v)_{j k}$ by

and

$$
\begin{aligned}
a(\boldsymbol{v})_{j k} & =\left(\underset{v}{\stackrel{\mathcal{L}}{u}} \boldsymbol{\varphi}_{j}{ }^{l}\right) \boldsymbol{\varphi}_{l k} \\
& =\boldsymbol{v}^{r}\left(\nabla_{r} \boldsymbol{\varphi}_{j}{ }^{l}\right) \boldsymbol{\varphi}_{l k}-\boldsymbol{\varphi}_{j}^{r}\left(\nabla_{r} v^{l}\right) \boldsymbol{\varphi}_{l k}-\nabla_{j} \boldsymbol{v}_{k}
\end{aligned}
$$

respectively.

$$
\begin{aligned}
b(\boldsymbol{v})_{j k} & =\left(\boldsymbol{v}^{r} \nabla_{r} \boldsymbol{\varphi}_{j}{ }^{l}+\boldsymbol{\varphi}_{r}{ }^{l} \nabla_{j} \boldsymbol{v}^{r}+\boldsymbol{\phi}_{j}^{r} \nabla_{r} \boldsymbol{v}^{l}\right) \boldsymbol{\varphi}_{l k} \\
& =\boldsymbol{v}^{r}\left(\nabla_{r} \boldsymbol{\varphi}_{j}{ }^{l}\right) \boldsymbol{\varphi}_{l k}+\boldsymbol{\varphi}_{j}^{r}\left(\nabla_{r} \boldsymbol{v}^{l}\right) \boldsymbol{\varphi}_{l k}-\nabla_{j} \boldsymbol{v}_{k}
\end{aligned}
$$

We notice that $a(v)_{j k}=0$ resp. $b(v)_{j k}=0$ is equivalent to the fact that the vector field $v$ is analytic resp. covariant analytic.

In the following we denote $a_{j k}$ resp. $b_{j k}$ instead of $a(v)_{j k}$ resp. $b(v)_{j k}$ for simplicity. We shall put

$$
a^{2}(v)=a_{j k} a^{j k}, \quad b^{2}(v)=b_{j k} b^{j k}
$$

and compute these. By definition (4.1) we have

$$
a^{2}(v)=\left[\boldsymbol{v}^{r}\left(\nabla_{r} \boldsymbol{\varphi}_{j}{ }^{l}\right) \boldsymbol{\varphi}_{l k}-\boldsymbol{\varphi}_{j}{ }^{r}\left(\nabla_{r} \boldsymbol{v}^{l}\right) \boldsymbol{\varphi}_{l k}-\nabla_{j} \boldsymbol{v}_{k}\right] \times
$$

16) For pseudo-Kählerian case, see Yano, K. [7], p. 238. 


$$
\left[\boldsymbol{v}^{s}\left(\nabla_{s} \boldsymbol{\varphi}^{j p}\right) \boldsymbol{\varphi}_{p}{ }^{k}-\boldsymbol{\varphi}^{j s}\left(\nabla_{s} v^{p}\right) \boldsymbol{\varphi}_{p}{ }^{k}-\nabla^{j} \boldsymbol{v}^{k}\right]
$$

whose right hand member is the sum of the following nine terms $A_{1}$, $A_{2}, \ldots \ldots, C_{3}$.

$$
\begin{aligned}
& A_{1}=\boldsymbol{v}^{r}\left(\nabla_{r} \boldsymbol{\varphi}_{j}{ }^{l}\right) \boldsymbol{\varphi}_{l k} \boldsymbol{v}^{s}\left(\nabla_{s} \boldsymbol{\varphi}^{j p}\right) \boldsymbol{\varphi}_{p}{ }^{k}=-2 \boldsymbol{v}^{r} \boldsymbol{v}^{s}\left(\nabla_{s} \boldsymbol{\varphi}^{j p}\right) \nabla_{j} \boldsymbol{\varphi}_{p r}, \\
& A_{2}=-\boldsymbol{v}^{r}\left(\nabla_{r} \boldsymbol{\varphi}_{j}{ }^{l}\right) \boldsymbol{\varphi}_{l k} \boldsymbol{\varphi}^{j s}\left(\nabla_{s} \boldsymbol{v}^{p}\right) \boldsymbol{\varphi}_{p}{ }^{k}=-\boldsymbol{v}^{r}\left(\nabla_{r} \boldsymbol{\phi}_{j p}\right) \boldsymbol{\phi}^{j s} \nabla_{s} \boldsymbol{v}^{p}, \\
& A_{3}=-\boldsymbol{v}^{r}\left(\nabla_{r} \boldsymbol{\varphi}_{j}{ }^{l}\right) \boldsymbol{\varphi}_{l k} \nabla^{j} v^{k}=A_{2} \text {, } \\
& B_{1}=-\boldsymbol{\varphi}_{j}{ }^{r}\left(\nabla_{r} v^{l}\right) \boldsymbol{\varphi}_{l k} v^{s}\left(\nabla_{s} \boldsymbol{\varphi}^{j p}\right) \boldsymbol{\varphi}_{p}{ }^{k}=A_{2} \text {, } \\
& B_{2}=\boldsymbol{\varphi}_{j}^{r}\left(\nabla_{r} v^{l}\right) \boldsymbol{\varphi}_{l k} \boldsymbol{\varphi}^{j s}\left(\nabla_{s} v^{p}\right) \boldsymbol{\varphi}_{p}{ }^{k}=\left(\nabla^{r} v^{s}\right) \nabla_{r} v_{s} \text {, } \\
& B_{3}=\boldsymbol{\varphi}_{j}^{r}\left(\nabla_{r} v^{l}\right) \boldsymbol{\varphi}_{l k} \nabla^{j} \boldsymbol{v}^{k}=-\boldsymbol{\varphi}^{r_{j}} \boldsymbol{\phi}^{l k}\left(\nabla_{r} v_{l}\right) \nabla_{j} v_{k} \text {, } \\
& C_{1}=-\boldsymbol{v}^{s}\left(\nabla_{s} \boldsymbol{\phi}^{j p}\right) \boldsymbol{\phi}_{p}{ }^{k} \nabla_{j} \boldsymbol{v}_{k}=A_{2} \text {, } \\
& C_{2}=\phi^{j s}\left(\nabla_{s} v^{p}\right) \boldsymbol{\varphi}_{p}{ }^{k} \nabla_{j} v_{k}=B_{3} \text {, } \\
& C_{3}=\left(\nabla_{j} v_{k}\right) \nabla^{j} v^{k}=B_{2} \text {. }
\end{aligned}
$$

Hence $a^{2}(v)=A_{1}+4 A_{2}+2 B_{2}+2 B_{3}$, so we obtain the following formula :

$$
\begin{aligned}
\frac{1}{2} a^{2}(\boldsymbol{v})= & -\boldsymbol{v}^{s} \boldsymbol{v}^{r}\left(\nabla_{s} \boldsymbol{\varphi}^{j p}\right) \nabla_{j} \boldsymbol{\varphi}_{p r}-2 \boldsymbol{v}^{r}\left(\nabla_{r} \boldsymbol{\varphi}_{j p}\right) \boldsymbol{\varphi}^{j s} \nabla_{s} \boldsymbol{v}^{p} \\
& -\boldsymbol{\varphi}^{r^{j}} \boldsymbol{\varphi}^{l k}\left(\nabla_{r} \boldsymbol{v}_{l}\right) \nabla_{j} \boldsymbol{v}_{k}+\left(\nabla_{j} \boldsymbol{v}_{k}\right) \nabla^{j} \boldsymbol{v}^{k} .
\end{aligned}
$$

For $b^{2}(v)$, on taking account of the similarity of (5.1) and (5.2), we have

$$
b^{2}(v)=A_{1}+2 B_{2}-2 B_{3}
$$

from which we see that

$$
\begin{aligned}
\frac{1}{2} b^{2}(v)= & -v^{s} v^{r}\left(\nabla_{s} \varphi^{j p}\right) \nabla_{j} \boldsymbol{\varphi}_{p r} \\
& +\boldsymbol{\varphi}^{r_{j}} \boldsymbol{\varphi}^{l k}\left(\nabla_{r} \boldsymbol{v}_{l}\right) \nabla_{j} \boldsymbol{v}_{k}+\left(\nabla_{j} \boldsymbol{v}_{k}\right) \nabla^{j} \boldsymbol{v}^{k} .
\end{aligned}
$$

In the next place we shall compute $\nabla^{j} a_{j k}$ which is the sum of the following six terms $a_{1}, \ldots \ldots \ldots, a_{6}$.

$$
\begin{aligned}
& a_{1}=\left(\nabla^{j} \boldsymbol{v}^{r}\right)\left(\nabla_{r} \boldsymbol{\varphi}_{j}{ }^{l}\right) \boldsymbol{\varphi}_{l k}=-\left(\nabla^{j} \boldsymbol{v}^{r}\right) \boldsymbol{\varphi}_{j}{ }^{l} \nabla_{r} \boldsymbol{\varphi}_{l k}, \\
& a_{2}=v^{r}\left(\nabla^{j} \nabla_{j} \boldsymbol{\varphi}_{j}{ }^{l}\right) \boldsymbol{\phi}_{l k}=v^{r} R_{r k}^{*}-\boldsymbol{v}^{r} R_{r k}, \\
& a_{3}=v^{r}\left(\nabla_{r} \boldsymbol{\varphi}_{j}^{l}\right) \nabla^{j} \boldsymbol{\varphi}_{l k}, \\
& a_{4}=-\boldsymbol{\phi}_{j}^{r}\left(\nabla^{j} \nabla_{r} v \boldsymbol{\phi}_{j}{ }^{l}\right) \boldsymbol{\phi}_{l k}=-v^{r} R_{r k}^{*}, \\
& a_{5}=-\left(\nabla_{r} v^{l}\right) \boldsymbol{\varphi}_{j}^{r} \nabla^{j} \boldsymbol{\varphi}_{l k}=\left(\nabla^{j} \boldsymbol{v}^{r}\right) \boldsymbol{\varphi}_{j}{ }^{l} \nabla_{l} \boldsymbol{\varphi}_{r k}, \\
& a_{6}=-\nabla^{j} \nabla_{j} v_{k} \text {. }
\end{aligned}
$$

Consequently, by virtue of 


$$
\begin{aligned}
& a_{1}+a_{5}=\left(\nabla^{j} v^{r}\right) \boldsymbol{\varphi}_{j}^{l} \nabla_{k} \boldsymbol{\varphi}_{r l}, \\
& a_{2}+a_{4}=-v^{r} R_{r k},
\end{aligned}
$$

we have

(5.5) $\quad \nabla^{j} a_{j k}=-\left(\nabla^{r} \nabla_{r} \boldsymbol{v}_{k}+\boldsymbol{v}^{r} R_{r k}\right)+\boldsymbol{v}^{r}\left(\nabla_{r} \boldsymbol{\varphi}_{j}{ }^{l}\right) \nabla^{j} \boldsymbol{\varphi}_{l k}+\left(\nabla^{j} \boldsymbol{v}^{r}\right) \boldsymbol{\varphi}_{j}{ }^{l} \nabla_{k} \boldsymbol{\varphi}_{r l}$.

For $\nabla^{j} b_{j k}$, using the above notation, we have

$$
\nabla^{j} b_{j k}=a_{1}+a_{2}+a_{3}-a_{4}-a_{5}+a_{6} .
$$

As the relations

$$
\begin{aligned}
& a_{2}-a_{4}=2 v^{r} R_{r k}^{*}-v^{r} R_{r k}, \\
& a_{1}-a_{5}=-\left(\nabla^{j} v^{r}\right) \boldsymbol{\varphi}_{j}{ }^{l}\left(\nabla_{r} \boldsymbol{\varphi}_{l k}+\nabla_{l} \boldsymbol{\varphi}_{r k}\right)
\end{aligned}
$$

hold good, it follows that

(5. 6) $\quad \nabla^{j} b_{j k}=-\left(\nabla^{r} \nabla r v_{k}+v^{r} R_{r k}\right)+2 v^{r} R_{r k}^{*}+v^{r}\left(\nabla_{r} \boldsymbol{\varphi}_{j}^{l}\right) \nabla^{j} \boldsymbol{\varphi}_{l k}$

$$
-\left(\nabla^{j} \boldsymbol{v}^{r}\right) \boldsymbol{\varphi}_{j}{ }^{l}\left(\nabla_{r} \cdot \boldsymbol{\varphi}_{l k}+\nabla_{l} \boldsymbol{\varphi}_{r k}\right)
$$

We next substitute (5.1) and (5.5) in the equation

$$
\nabla^{j}\left(a_{j k} v^{k}\right)=v^{k} \nabla^{j} a_{j k}+a_{j k} \nabla^{j} v^{k},
$$

then we get

$$
\begin{aligned}
\nabla^{j}\left(a_{j k} v^{k}\right)= & -\left(\nabla^{r} \nabla_{r} v_{k}+v^{r} R_{r k}\right) v^{k}+v^{r} v^{k}\left(\nabla_{r} \boldsymbol{\varphi}_{j}{ }^{l}\right) \nabla_{j} \boldsymbol{\varphi}_{l k} \\
& +2\left(\nabla^{j} v^{r}\right) \boldsymbol{\phi}_{j}{ }^{l} v^{k} \nabla_{k} \boldsymbol{\varphi}_{r l}+\boldsymbol{\varphi}^{r j} \boldsymbol{\rho}^{l k}\left(\nabla_{r} \boldsymbol{v}_{l}\right) \nabla_{j} \boldsymbol{v}_{k} \\
& -\left(\nabla_{j} v_{k}\right) \nabla^{j} v^{k} .
\end{aligned}
$$

From (5.3) and (5.7), we get

$$
\frac{1}{2} a^{2}(v)+\nabla^{j}\left(a_{j k} v^{k}\right)=-\left(\nabla^{r} \nabla_{r} v_{i}+v^{r} R_{r i}\right) v^{i} .
$$

Hence, by Green's theorem, we obtain the following

THEOREM 5.1. ${ }^{17)}$ In a compact almost-Kählerian manifold $M$, the integ. ral formula

$$
\int_{M}\left[\left(\nabla^{r} \nabla_{r} v_{i}+v^{r} R_{r i}\right) v^{i}+\frac{1}{2} a^{2}(v)\right] d \sigma=0
$$

is valid for any vector field $v$, where

$$
a^{2}(v)=a_{j k} a^{j k}, a_{j k}=\left(\underset{v}{\stackrel{\circ}{L}} \boldsymbol{\varphi}_{j}^{l}\right) \boldsymbol{\varphi}_{l k} .
$$

We next substitute (5.2) and (5.6) in

$$
\nabla^{j}\left(b_{j k} v^{k}\right)=v^{k} \nabla^{j} b_{j k}+b_{j k} \nabla^{j} v^{k}
$$

and obtain the formula

$$
\nabla^{j}\left(b_{j k} v^{k}\right)=-\left(\nabla^{r} \nabla r v_{k}+v^{r} R_{r k}\right) v^{k}+R_{r k}^{*} v^{r} v^{k}
$$

17) For pseudo-Kählerian case, see Yano, K. [7], p. 278, Lichnerowicz, A. [3]. 


$$
\begin{aligned}
& +\boldsymbol{v}^{r} \boldsymbol{v}^{k}\left(\nabla_{r} \boldsymbol{\varphi}_{j}{ }^{l}\right) \nabla_{j} \boldsymbol{\varphi}_{l k}-2\left(\nabla^{j} \boldsymbol{v}^{r}\right) \boldsymbol{\varphi}_{j}{ }^{l}\left(\nabla_{r} \boldsymbol{\varphi}_{l k}\right) \boldsymbol{v}^{k} \\
& -\boldsymbol{\phi}^{r_{j}} \boldsymbol{\phi}^{l k}\left(\nabla_{r} \boldsymbol{v}_{l}\right) \nabla_{j} \boldsymbol{v}_{k}-\left(\nabla_{j} \boldsymbol{v}_{k}\right) \nabla^{j} \boldsymbol{v}^{k} .
\end{aligned}
$$

Hence from (5.4) and (5.10), it follows that

$$
\begin{aligned}
\nabla^{j}\left(b_{j k} v^{k}\right) & +\frac{1}{2} b^{2}(v)=-\left(\nabla^{r} \nabla_{r} v_{i}+v^{r} R_{r i}\right) v^{i} \\
& +R_{r i}^{*} v^{r} v^{i}-2 \nabla^{j} v^{r}\left(\nabla_{r} \boldsymbol{\varphi}_{j l}\right) \boldsymbol{\varphi}_{k}{ }^{l} v^{k} .
\end{aligned}
$$

On the other hand, (2.11) holds good for any vector field $v$. Hence subtructing (5.11) from (2.11) and dividing by 2 , we have

$$
\begin{aligned}
& \frac{1}{2}\left\{\left(\nabla^{r} \nabla_{r} \widetilde{v_{i}}-\widetilde{v}^{r} R_{r i} \tilde{v^{i}}-\nabla^{j}\left(b_{j k} v^{k}\right)\right\}\right. \\
& =\left(\nabla^{r} \nabla_{r} v_{i}+v^{r} R_{r i}\right) v^{i}-2 R_{r i}^{*} v^{r} v^{i}+\nabla^{j} v^{r}\left(\nabla_{j} \boldsymbol{\varphi}_{r l}\right. \\
& \left.+\nabla_{\boldsymbol{r}} \boldsymbol{\varphi}_{j l}\right) \boldsymbol{\varphi}_{i}{ }^{l} \boldsymbol{v}^{i}+\frac{1}{4} b^{2}(v) \text {. }
\end{aligned}
$$

If we apply Lemma 2.1 to $\widetilde{v}$, we have in compact $M$

$$
\int_{M}\left(\nabla^{r} \nabla_{r} \widetilde{v}_{i}-R_{r i} \widetilde{v^{r}}\right) \widetilde{v}^{i} d \sigma=-\int_{M} S(\widetilde{v}) d \sigma
$$

so integrating (5.12) we obtain the following

THEOREM 5.2. In a compact almost-Kählerian manifold $M$, the integral formula

$$
\begin{gathered}
\int_{M}\left[\left(\nabla^{r} \nabla_{r} v_{i}+R_{r i} v^{r}-2 R_{r i}^{*} v^{r}\right) v^{i}+\nabla^{j} v^{r}\left(\nabla_{j} \boldsymbol{\varphi}_{r i}+\nabla_{r} \boldsymbol{\varphi}_{j i}\right) \boldsymbol{\varphi}_{t}{ }^{i} \boldsymbol{v}^{t}\right. \\
\left.+\frac{1}{4} b^{2}(\boldsymbol{v})+\frac{1}{2} S(\widetilde{v})\right] d \sigma=0
\end{gathered}
$$

is valid for any vector field $v$, where

$$
\begin{aligned}
& b^{2}(v)=b_{j k} b^{j k}, \widetilde{v_{i}}=\phi_{i}{ }^{t} v_{t}, \\
& b_{j k}=\left(v^{r} \nabla_{r} \boldsymbol{\varphi}_{j}{ }^{l}+\boldsymbol{\varphi}_{r}{ }^{l} \nabla_{j} v^{r}+\boldsymbol{\varphi}_{j}^{r} \nabla_{r} v^{l}\right) \boldsymbol{\varphi}_{l k} \text {, } \\
& S(\widetilde{v})=\frac{1}{2}\left(\nabla^{r} \widetilde{v^{s}}-\nabla^{s} \widetilde{v^{r}}\right)\left(\nabla_{r} \widetilde{v_{s}}-\nabla_{s} \widetilde{v_{r}}\right)+\left(\nabla^{r} \widetilde{v_{r}}\right)\left(\nabla^{s} \widetilde{v_{s}}\right) .
\end{aligned}
$$

6. Theorems. In this section we shall consider a compact almost-Kählerian manifold $M$ and obtain several theorems. In $\$ 3$, we have proved that an analytic vector $v$ satisfies the equation (3.7) i. e.

$$
\nabla^{r} \nabla_{r} v_{i}+R_{r i} v^{r}=0 .
$$

In a compact manifold, we know from Theorem 5.1 that (6.1) is also sufficient for the analyticity of $v$. 
THEOREM 6. 1. ${ }^{18)}$ In a compact almost-Kählerian manifold, a necessary and sufficient condition in order that a vector $v$ be an analytic vector is that

$$
\nabla^{r} \nabla r v_{i}+R_{r i} v^{r}=0 .
$$

By virtue of Lemma 2.2, it follows the following

COROLLARY. ${ }^{19)}$ In a compact almost-Kählerian manifold, any Killing vector is an analytic vector.

COROLLARY. ${ }^{20)}$ In a compact almost-Kählerian manifold, a necessary and sufficient condition in order that an analytic vector $v$ be a Killing vector is $\nabla_{r} v^{r}=0$.

Consider an analytic vector $v$, then from (3.6), (6.1) and Theorem 5.2, we get

$$
\int_{\boldsymbol{M}}\left(R_{r i}^{*} \boldsymbol{v}^{r} \boldsymbol{v}^{i}\right) d \sigma=\int_{M}\left[\frac{1}{4} b^{2}(v)+\frac{1}{2} S(\widetilde{v})\right] d \sigma \geqq 0 .
$$

Hence we have, on taking account of Theorem 4.7,

THEOREM 6. 2. In a compact almost-Kählerian manifold $M$, the integral

$$
J(v)=\int_{M}\left(R_{r i}^{*} v^{r} v^{i}\right) d \sigma
$$

is positive or zero for any analytic vector $v$. If $J(v)=0$ for an analytic vector $v$, then the vector $v$ is a parallel vector field. that is

Now suppose that the manifold $M$ is a space of constant curvature,

$$
R_{k j i}{ }^{h}=k\left(g_{k i} \delta_{j}^{h}-g_{j i} \delta_{k}^{h}\right), \quad \text { where } \quad k=-\frac{R}{n(n-1)},
$$

holds good. Then we have $R_{j i}^{*}=-2 k g_{j i}$, from which it follows the following

COROLLARY. ${ }^{21)}$ In a compact almost-Kählerian manifold of constant curvature, there does not exist a non-trivial analytic vector field if $R<0$.

Let $v$ be a conformal Killing vector, then from Lemma 2.3 we have

$$
\int_{M}\left(\nabla^{r} \nabla_{r} v_{i}+v^{r} R_{r i}\right) v^{i} d \sigma=-\frac{n-2}{n} \int_{M} v^{i} \nabla i \nabla r v^{r} d \sigma .
$$

18) For pseudo-Kählerian case, Yano, K. [7], p. 280.

19) Apte, M. [1].

20) For pseudo-Kählerian case, Yano, K. [7], p. 281.

21) We remark that there does not exist a compact almost-Kählerian manifold of constant curvature with $R>0$. cf. Yano, K. and S. Bochner, [8], p. 80. 
On the other hand, integrating

$$
\nabla_{i}\left(v^{i} \nabla_{r} v^{r}\right)=v^{i} \nabla_{i} \nabla_{r} v^{r}+\left(\nabla_{i} v^{i}\right) \nabla_{r} v^{r}
$$

over $M$, we get

$$
-\int_{M} v^{i} \nabla i \nabla_{r} v^{r} d \sigma=\int_{M}\left(\nabla_{r} v^{r}\right)^{2} d \sigma .
$$

Hence, the equation

$$
\int_{M}\left(\nabla^{r} \nabla_{r} v_{i}+R_{r i} v^{r}\right) v^{i} d \sigma=\frac{n-2}{n} \int_{M}\left(\nabla_{r} v^{r}\right)^{2} d \sigma
$$

holds good. Therefore by virtue of Theorem 5.1, we obtain

$$
\int_{M}\left[\frac{n-2}{n}\left(\nabla_{r} v^{r}\right)^{2}+\frac{1}{2} a^{2}(v)\right] d \sigma=0,
$$

from which, taking account of Lemma 2.2, we find the following

THEOREM 6.3. ${ }^{22)}$ In a compact almost-Kählerian manifold $M$, any conformal Killing vector is a Killing vector if $n>2$ and an analytic vector for $n=2$.

Let $v$ ke a projective Killing vector, then the equation (2.9) i. e.

$$
\nabla^{r} \nabla_{r} v_{i}+R_{r i} v^{r}=\frac{2}{n+1} \nabla_{i} \nabla_{r} v^{r}
$$

holds. Hence by virture of Theorem 5.1 and (6.2), we have the following integral formula

$$
\frac{2}{n+1} \int_{M}\left(\nabla_{r} v^{r}\right)^{2} d \sigma=\frac{1}{2} \int_{M} a^{2}(v) d \sigma .
$$

Thereby we get

THEOREM 6. 4. In a compact almost-Kählerian manifold, if an analytic vector is at the same time a projective Killing vector, then it is a Killing vector.

In a compact pseudo-Kählerian manifold, if $v$ is harmonic, then $\tilde{v}$ is also harmonic. In our case, the same argument does not hold.

A covariant analytic vector $v$ satisfies

$$
\nabla^{r} \nabla_{r} \boldsymbol{v}_{i}+R_{r i} \boldsymbol{v}^{r}-R_{r i}^{*} \boldsymbol{v}^{r}+\nabla^{j} \boldsymbol{v}^{r}\left(\nabla_{j} \boldsymbol{\varphi}_{r t}+\nabla_{r} \boldsymbol{\Phi}_{j t}\right) \boldsymbol{\varphi}_{i}{ }^{t}=0
$$

by virtue of (4.10). Conversely, in compact $M$, if a vector field $v$ satisfies (6.3), Theorem (5.2) asserts that $b^{2}(v)=0$ and $S(\widetilde{v})=0 . b^{2}(v)$ means that the vector $v$ is covariant analytic and $S(\tilde{v})=0$ means that the vector $\tilde{v}$ is harmonic. Then from Theorem 4.4, since $\tilde{v}$ is also a covariant analytic vector, the

22) For Kählerian case, Lichnerowicz, A. [3]. 
same argument is applicable to $\tilde{v}$, so $v$ is also harmonic. Consequently we find

THEOREM 6.5. In a compact almost-Kählerian manifold, a necessary and sufficient condition in order that a vector field $v$ be covariant analytic is that $v$ satisfies (6. 3).

THEOREM 6.6. In a compact almost-Kählerian manifold, a necessary and sufficient condition in order that $a$ vector $v$ be covariant analytic is that $v$ and $\widetilde{v}$ are both harmonic.

7. Lie algebra of analytic vectors in a compact almost-KählerEinstein manifold. In this section we shall consider a compact almostKähler-Einstein manifold $M$ and give a theorem on the Lie algebra of analytic vectors on $M$. This theorem corresponds to Matsushima's theorem ${ }^{23)}$ in a compact Kähler-Einstein manifold and similar to Lichnerowicz' theorem ${ }^{24}$ ) on the Lie alge ra of conformal Killing vectors in a compact Einstein marifold.

As $M$ is an Einstein manifold, it holds that

$$
R_{j i}=c g_{j i}, \quad c=\frac{R}{n} .
$$

We shall assume that $c \neq 0$. In this case, (6.1) reduces to

$$
\nabla^{r} \nabla_{r} v_{i}+c v_{i}=0
$$

from which we have

$$
\nabla^{r} \nabla_{r} \nabla^{i} v_{i}+2 c \nabla^{i} v_{i}=0
$$

or if we put $f=\nabla^{i} v_{i}$, we have

$$
\nabla^{r} \nabla r f+2 c f=0 .
$$

If a scalar function $f$ is a solution of (7.2), the equation

$$
\nabla^{r} \nabla_{r} \nabla_{i} f+c \nabla_{i} f=0
$$

is valid. Hence the gradient $\nabla_{i} f$ of $f=\nabla_{i} v^{i}$ for an analytic vector $v$ is also an analytic vector.

Now we put

$$
p_{i}=v_{i}+\frac{1}{2 c} \nabla_{i} f, \quad f=\nabla_{i} v^{i}
$$

then from (7.2) we have $\nabla^{i} p_{i}=0$. Hence by virtue of the Corollary of Theorem 6.1, $p_{i}$ is a Killing vector. From (7.4), we have

23) Matsushima, Y. [4]. Yano, K. [7]. p. 285.

24) Lichnerowicz, A. [2]. Yano, K. [7], p. 276. 


$$
v_{i}=p_{i}-\frac{1}{2 c} \nabla_{i} f
$$

Conversely, if $p_{i}$ is a Killing vector and $f$ is a solution of (7.2). then the vector $v$ defined by $(7.5)$ is an analytic vector. We shall denote by $L$ resp. $L_{1}$ the Lie alge'ra of analytic resp. Killing vectors and by $L_{2}$ the vector space of the gradient of solutions of (7.2). Then the above argument asserts that $L=L_{1}+L_{2}$, where + means a direct sum. After some simple calculation, we have the relation

$$
\left[L_{1}, L_{2}\right] \subset L_{2}, \quad\left[L_{2}, L_{2}\right] \subset L_{1} .
$$

Hence we get the following

THEOREM 7.1. In a compact almost-Kähler-Einstein $(R \neq 0)$ manifold $M$, the direct sum

$$
L=L_{1}+L_{2}
$$

is valid, where $L$ resp. $L_{1}$ is the Lie algebra of analytic resp. Killing vectors on $M$ and $L_{2}$ is the vector space consisting of the gradient of solutions of

$$
\nabla^{r} \nabla_{r} f+2 c f=0, \quad c=\frac{R}{n} .
$$

In this decomposition, the relations

$$
\left[L_{1}, L_{2}\right] \subset L_{2}, \quad\left[L_{2}, L_{2}\right] \subset L_{1}
$$

hold.

Now let $v$ be an analytic vector in a compact almost-Kählerian manifold $M$. Then $v$ satisfies the equation

$$
\nabla^{r} \nabla_{r} v_{i}+R_{r i} v^{r}=0
$$

Hence we get easily

$$
\frac{1}{2} \nabla^{r} \nabla_{r}\left(v_{i} v^{i}\right)=\left(\nabla_{r} v_{i}\right)\left(\nabla^{r} v^{i}\right)-R_{r i} v^{r} v^{i}
$$

Integrating the last eqation over $M$, we have

THEOREM 7.2. In a compact almost-Kählerian manifold $M$, the integral

$$
\int_{M}\left(R_{r i} v^{r} v^{i}\right) d \sigma
$$

is positive or zero for an analytic vector $v$.

COROLLARY. In a compact almost-Kähler-Einstein manifold with scalar curvature $R<0$, there does not exist a non-trivial analytic vector.

From this corollary we have again the corollary of Theorem 6.2.

We remark here the following facts. In theorems in this section the one 
which plays the essential role is not the almost-complex structure but the equation (7.1).

8. Canonical connection. In an almost-Hermitian manifold, if we put

$$
\Gamma_{j i}^{h}=\left\{\begin{array}{l}
h \\
j i
\end{array}\right\}-\frac{1}{2} \boldsymbol{\varphi}_{r}{ }^{h} \nabla_{j} \boldsymbol{\varphi}_{i}{ }^{r},
$$

then tensors $g_{j \imath}$ and $\boldsymbol{\varphi}_{j}{ }^{i}$ are both covariantly constant with respect to the affine connection $\Gamma_{j i}^{h}$. There are many affine connections with this property, but we shall call $\Gamma_{j i}^{h}$ defined by (8.1) the canonical connection. In this section we shall calculate the curvature tensor of the canonical connection and obtain certain identities which are useful in the next section. Consider the canonical connection (8.1) in our almost-Kählerian manifold and denote by $K_{k j i}{ }^{h}$ the curvature tensor. If we put

$$
t_{k i}{ }^{h}=-\frac{1}{2} \boldsymbol{\varphi}_{p}{ }^{h} \nabla_{k} \boldsymbol{\varphi}_{i}{ }^{p}
$$

and substitute in the identity

$$
K_{k j i}{ }^{h}=R_{k j i}{ }^{h}+\nabla_{k} t_{j i}{ }^{h}-\nabla_{j} t_{k i}{ }^{h}+t_{k r}{ }^{h} t_{j i}{ }^{r}-t_{j r}{ }^{h} t_{k i}{ }^{r},
$$

then we find, after some calculation,

$$
K_{k j i}{ }^{h}=\frac{1}{2}\left(R_{k j i}{ }^{h}-R_{k j p}{ }^{r} \boldsymbol{\varphi}_{i}{ }^{p} \boldsymbol{\varphi}_{r}{ }^{h}\right)-\frac{1}{4}\left\{\left(\nabla_{k} \boldsymbol{\varphi}_{r}{ }^{h}\right) \nabla_{j} \boldsymbol{\varphi}_{i}{ }^{r}-\left(\nabla_{j} \boldsymbol{\varphi}_{r}{ }^{h}\right) \nabla_{k} \boldsymbol{\varphi}_{i}{ }^{r}\right\} .
$$

Transvecting (8.2) with ${\varphi_{h}}^{i}$, we get

$$
K_{k j i}{ }^{h} \boldsymbol{\varphi}_{h}{ }^{i}=R_{k j i}{ }^{h} \boldsymbol{\varphi}_{h}{ }^{i}-\frac{1}{2}\left(\nabla_{k} \boldsymbol{\varphi}_{r}{ }^{h}\right)\left(\nabla_{j} \boldsymbol{\varphi}_{i}{ }^{r}\right) \boldsymbol{\varphi}_{h}{ }^{i},
$$

from which it follows that

$$
K_{h l}^{*}=R_{h l}^{*}-\frac{1}{4}\left(\nabla_{k} \boldsymbol{\varphi}_{p q}\right) \nabla_{l} \boldsymbol{\varphi}^{p q},
$$

where

Now we put

$$
K_{h l}^{*}=\frac{1}{2} K_{k i p}{ }^{q} \boldsymbol{\varphi}_{q}{ }^{p} \boldsymbol{\varphi}_{l}{ }^{i}
$$

$$
T_{k j}=-\left(\nabla_{k} \boldsymbol{\varphi}_{r}{ }^{h}\right)\left(\nabla_{j} \boldsymbol{\varphi}_{i}{ }^{r}\right) \boldsymbol{\varphi}_{h}{ }^{i},
$$

and shall calculate $T_{j k} v^{k}$ for any vector feld $v$. On taking account of (1.4) we have

$$
\begin{aligned}
-\left(\nabla_{k} \boldsymbol{\varphi}_{r h}\right) v^{k} & =\left(\nabla_{r} \boldsymbol{\varphi}_{h k}-\nabla_{h} \boldsymbol{\varphi}_{r k}\right) v^{k} \\
& =\nabla_{r} \tilde{v}_{h}-\nabla_{h} \widetilde{v}_{r}+\boldsymbol{\varphi}_{r k} \nabla_{h} v^{k}-\boldsymbol{\varphi}_{h k} \nabla_{r} v^{k}
\end{aligned}
$$

Hence it holds that

$$
T_{j k} v^{k}=\left(\nabla_{r} \widetilde{v}_{h}-\nabla_{h} \widetilde{v_{r}}\right)\left(\nabla_{j} \boldsymbol{\varphi}_{p}^{r}\right) \boldsymbol{\varphi}^{h p}+2\left(\nabla^{p} v^{q}\right) \nabla_{j} \boldsymbol{\varphi}_{p q} .
$$


Let $\boldsymbol{v}$ be an analytic vector, then by virtue of (3.3), we have

$$
\left(\nabla_{r} \widetilde{v_{h}}-\nabla_{h} \widetilde{v_{r}}\right)\left(\nabla_{j} \varphi_{p}{ }^{r}\right) \boldsymbol{\varphi}^{h p}=-\nabla_{j} \varphi^{h r}\left(\nabla_{h} v_{r}+\nabla_{r} v_{h}\right)=0 .
$$

Therefore, for an analytic vector $v$ we have the following formulas

$$
v^{k} K_{j k}^{*}=v^{k} R_{j k}^{*}+\frac{1}{2} \nabla^{p} v^{q}\left(\nabla_{l} \boldsymbol{\varphi}_{p q}\right) \boldsymbol{\varphi}_{j}{ }^{l} .
$$

9. A generalization of Apte's theorem. Let $v$ he an analytic vector in a compact almost-Kählerian manifold $M$. Then, from (3.6) and lemma 2.4 we have

$$
\int_{M}\left[R_{k j}^{*} v^{k} v^{j}+\nabla^{p} v^{q}\left(\nabla_{i} \boldsymbol{\varphi}_{p q}\right) \boldsymbol{\varphi}_{t}{ }^{i} v^{t}\right] d \sigma=\int_{M} S(\widetilde{\mathcal{V}}) d \sigma .
$$

On taking account of (8.5), we find

$$
\int_{M}\left(K_{k j}^{*} v^{k} v^{j}\right) d \sigma=\int_{M} S(\widetilde{v}) d \sigma .
$$

If $\tilde{S(\tilde{v})}=0$ holds, then $\tilde{v}$ is harmonic, kecause of (2.14). And in this case, $v$ is a Killing vector by virtue of (3.3). Hence we obtain the following theorem which is a generalization of Apte's theorem.

THEOREM 9.1. ${ }^{25)}$ In a compact almost-Kählerian manifold, the integral

$$
I(v)=\int_{s I}\left(K_{k j}^{*} v^{k} v^{j}\right) d \sigma
$$

is positive or zero for an analytic vector. If $I(v)=0$ for an analytic vector, then the vector $v$ is a Killing rector and $\tilde{v}$ is harmonic.

From the above theorem and (8.4), we have Theorem 6.2, again.

OCHANOMIZU UNIVERSITY, TOKYO.

\section{BIBLIOGRAPHY}

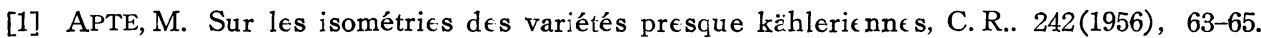

[2] LichNEROWICZ, A. Transformations infinitésimales conformes de certaines variétés riemanniennes compactes. C. R. Acad. Sci. Paris. 241(1955), 726-729.

[3] Lichnerowicz, A. Sur les transfor mations analytiçues des variétés kähleriennes compactes, C, R., 1957, 244(1957), 3011-3013.

[4] MAtsushima, Y. Sur la structure du groupe d'koméomorphismes analytiques d'une certaine variété kählerienne, Nagoya, 11 (1957) 145-150.

[5] SASAKI, S. AND K. YANO, Pseucuo-analytic veciois on pseućo-Këhlerian manifolds. Pacific Journal of Math. 5(1955), 987-993.

[6] Schouten, J. A. AND K. YANO, $\mathrm{C}$ invarient sulspaces in the alnost complex $X_{2} n$, Indagationes Math. (1955), 261-269.

[7] YANO, K. The theory of Lie derivatives and its applications, Amsterdam, 1955.

[8] YANO, K. AND S. BOChNER, Curvature and Betti numbeis, Princeion Univ. Press, 1958.

[9] YANO, K. AND I. MOGI, On real representations of Kählerian manifolds. Ann. of Math., 61(1955), 170-189.

25) For Killing vector, see ApteM. [1]. 